\title{
Working on Epidemiological Data: Problems Encountered and Lessons Learned
}

\author{
Giovanni Delli Zotti \\ University of Trieste, Italy \\ giovanni.dellizotti@dispes.units.it
}

\begin{abstract}
In addition to the epidemiological analysis of hospital admissions and outpatient services, the INTEGRA Project envisages the development of a sexual and reproductive health index that aims to identify situations at risk or which require 'targeted' interventions. This implies the need to identify suitable indicators, whose characteristics are described in the first part of the article, with reference to the specific needs of the INTEGRA Project. A discussion follows on the distinction between micro and macro level indicators and some examples are illustrated. Some problems found by analyzing the Hospital Discharge Cards are then described and some solutions are proposed. A final specific topic concerns the implications of the transition from a database having 'hospital demission' as unit of analysis to a data archive based on 'patient' as the unit of analysis.
\end{abstract}

\section{Introduction}

The INTEGRA Project, in addition to the epidemiological analysis of hospitalizations and outpatient services provided, foresees the development of a Sexual and Reproductive Health Index that aims to identify situations at risk or which require 'targeted' interventions. Being a 'health' index, high values would indicate the absence of criticality; on the contrary, low values would signal more or less severe problems that need to be removed. Alternatively, an index of 'pathology' might be developed; however, it is more effective to 'think positive' and, after all, 'health' is the word used in international documents, starting from The Beijing Declaration promoted by the United Nations in 1995. The Declaration included the recognition of the human rights of women as integral to the achievement of optimal health and well-being: 'The human rights of women include their right to have control over and decide freely and responsibly on matters related to their sexuality, including sexual and reproductive health, free of coercion, discrimination and violence. Equal relationships between women and men in matters of sexual relations and reproduction, including full respect for the integrity of the person, require 
mutual respect, consent and shared responsibility for sexual behavior and its consequences' $(1995$, p. 36).

Making an index means starting from a general concept to arrive at its 'measurement' at the end of a process that can be quite complex. Even if we succeed in defining the concept with some precision, the property to be measured remains relatively abstract until we move on to its concrete specification. The concept of 'health' is perhaps less abstract than others, but rather complex, because we can talk about health (sexual, reproductive or otherwise) only after verifying the absence of several situations that could undermine it, or the presence of factors that can positively affect it. In methodological terms, it is therefore necessary to identify suitable 'indicators' and to define them operationally, meaning that we must specify how we intend to measure them. The indicators this way become variables that need to be opportunely combined in order to obtain the foreseen index.

Using the term 'opportunely,' we intend to recall the fact that indicators overlap more or less broadly the semantic range of the concept/property that we intend to measure. It is therefore legitimate to attribute to the indicators/variables appropriate weights, trying to replicate their degree of validity. In fact, when choosing indicators validity is an important requisite, since we need indicators that clearly point at the property we aim at measuring (face validity, see Drost, 2011). When establishing how to translate each indicator into a variable, the most important characteristic to seek is instead reliability of the outcome of measurement.

Other criteria may also be relevant, such as those listed in a site dedicated to the construction of indicators for Family Planning/Reproductive Health (FP/RH) (measureevaluation.org):

- Relevance: there is a clear relationship between the indicator and FP/RH

- Accuracy: the indicator measures what it purports to measure

- Importance: the measurement captures something that 'makes a difference' in program effectiveness

- Usefulness: the results point to areas for improvement. Furthermore, the indicator captures information that helps move FP/RH strategies, priorities, or programming forward

- Feasibility: data can be obtained with reasonable and affordable effort

- Credibility: the indicator has been recommended - and is being used - by leading experts and organizations such as WHO, UNAIDS, USAID, and UNFPA

- Validity: to the extent possible, the indicator has been field-tested (in- 
cluding those measured in Demographic and Health Surveys (DHS) and Reproductive Health Surveys (RHS)) or used in practice ${ }^{1}$

- Distinctiveness: the indicator lacks redundancy and does not measure something already captured under other indicators.

- In addition, where indicators are presented as part of a set, this set should meet another criterion, namely that the overall set is coherent and balanced.

Authors also point out that the indicators presented and analyzed where harmonized with those already in use, giving high priority to indicators from key initiatives such as the Millennium Development Goals ${ }^{2}$ and USAID's Global Health Initiative $(\mathrm{GHI}){ }^{34}$

The recent Guide for Monitoring and Evaluating Population-Health-Environment Programs (Moreland \& Curran, 2018), insists that each indicator must be valid to accurately measure a behavior, practice or task, reliable (consistently measurable in the same way by different observers), precise and, of course, measurable, that is quantifiable using available tools and methods. Authors add that indicators should also be timely, thus providing measurement at intervals that are relevant and appropriate for program goals and activities, and programmatically important, that means linked to a public health impact or to achieving the objectives needed for impact. The last criterion, labeled in the list above as 'usefulness', needs to be stressed, being significantly important for the scope of INTEGRA Project. The project has in fact mainly application purposes, because the Index of Sexual and Reproductive Health (SR Index) 'will be used by health professionals when it comes to migrant women and will have the possibility of being transferred to different health facilities and to ensure holistic care, as it enhances both the medical and the social dimension.'

The World Health Organization (1997), sometimes uses different words for features already mentioned, but adds also something new in her description of the 'good indicator:'

- Ethical: Data must respect people's rights to confidentiality, freedom of

\footnotetext{
1 'Validity' is here meant in a more technical sense but, being attributed to indicators tested in the field, also in comparison with similar instruments, it seems to be more appropriate to refer to reliability (test-retest reliability).

${ }^{2}$ See http://www.un.org/millenniumgoals.

${ }^{3}$ See https://www.usaid.gov.

${ }^{4}$ This is quite relevant for the INTEGRA Project, since the 'new index' aims to be inserted among the other indexes used for the assessment of integration policies.
} 
choice in supplying information, and informed consent regarding the nature and implications of the data required.

- Useful: the indicator acts as a marker of progress toward improved reproductive health status or as a measure of progress toward specified process goals.

- Scientifically robust: the indicator should be a valid, specific, sensitive, and reliable reflection of what it purports to measure.

- Representative: the indicator must adequately encompass all the issues or population groups it is expected to cover.

- Understandable: the indicator should be simple to define and its value easy to interpret.

- Accessible: it uses data that are already available or are relatively easy to acquire by feasible methods that have been validated in field trials.

In addition, the Canadian International Development Agency (CIDA) (1996) recommends that good indicators have the following characteristics:

- Participatory: the indicator has been developed in a participatory fashion.

- Relevant: the indicator has been formulated at a level the user can understand and is relevant to the users' needs.

- Sex-disaggregated: data are collected so that analysis can be conducted separately for males and females, if appropriate.

- Qualitative or quantitative: data are either quantitative or qualitative, as appropriate to the objectives of the project.

Noting that these are the characteristics authoritatively identified, a researcher intending to collect original data is responsible for operating in a way to satisfy them; intending instead to use pre-existing data, he can only ascertain the existence of these characteristics. Finding any shortcoming, he may look for alternative sources and/or recommend enhancing accuracy in collecting the data. This is the case of the INTEGRA Project, since researchers have the opportunity to suggest improvements, having also the task of proposing guidelines.

\section{Micro and Macro Level Indicators}

When dealing with indicators and indexes, the level of measurement is another aspect that must be carefully considered: it is a matter of deciding whether indicators will be measured and/or used at an individual (micro) 
or collective (macro) level. ${ }^{5}$ The list of Recommended Sexual and Reproductive Health and Rights Indicators for Post-2015 Sustainable Development Goals (Guttmacher Institute, 2015, p. 3) provides examples of macro-level indicators and specifies whether indicators are available, or just 'aspirational' (Table 1). ${ }^{6}$

As can be easily understood from terms such as 'proportion,' 'percentage' or 'rate', many indicators are built by gathering information at the individual level, later aggregated to estimate the health status of categories or groups of people defined according to gender, social status, country or cultural origin, etc. In other instances, indicators genuinely refer to the 'macro' level, since they do not derive from the aggregation of individual data (it deals, for example, of legislation, policies, or cultural traditions of the countries considered).

Undoubtedly, one may use 'macro' health indices to attribute a presumptuous degree of health/risk to single women coming from, or belonging to, specific countries or categories.

However, it is necessary to underline that some indicators, designed to monitor sexual and reproductive health in different countries of the world, are not necessarily useful for evaluating the situation of immigrant women. For example, the Reproductive health indicators lists, among others, antenatal care coverage, births attended by skilled health personnel and availability of basic essential obstetric care are (World Health Organization, 2006). In the countries of origin, these services may be lacking but, when dealing with women and their health, once immigrated to Italy and Slovenia we can consider these services available. Therefore, instead of ascertaining the availability of such services, it will be necessary to check whether women use them, particularly in the case of antenatal assistance.

Furthermore, using macro indices is a rough approximation since every woman, while sharing a social or ethno-national belonging, and thus a generic level of sexual and reproductive health, bears a specific situation that can improve or completely nullify the level determined by macro indicators. It is therefore evident that the information gathered at the individual level, useful for the contribution they can make to the construction of macroindices, are essential for determining the sexual and reproductive health of single women who, in the case of the INTEGRA project, are mainly those that use the services offered by hospitals and outpatient facilities.

\footnotetext{
${ }^{5}$ For a preliminary approach, see (https://en.wikipedia.org/wiki/Level_of_analysis).

${ }^{6}$ By citing not yet available indicators, the list is particularly useful for the INTEGRA Project, as it suggests directions to which it would be advisable to proceed.
} 
Table 1 Recommended Sexual and Reproductive Health and Rights Indicators

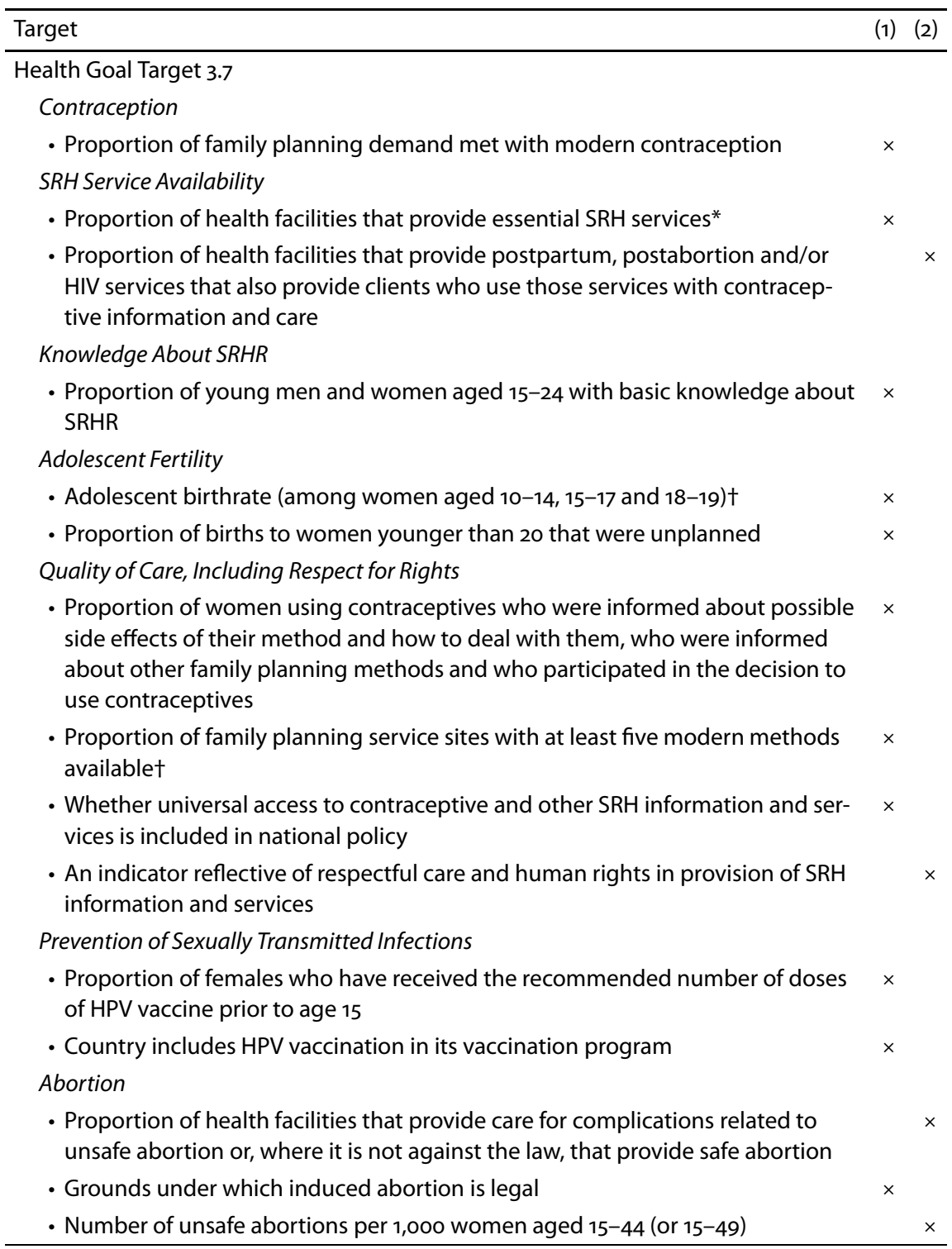

Continued on the following page

\section{The Hospital Demission Record (HDR)}

Having ascertained that many macro level indicators and indexes are already available, we will here focus on the micro level, dealing with the character- 
Table 1 Continued from the previous page

\begin{tabular}{lc}
\hline Target & (1) (2) \\
\hline Education Goal Target 4 & \\
- Comprehensive Sexuality Education & $\times$ \\
- Proportion of schools that serve students in the age range of $12-17$ years in & \\
$\quad$ which comprehensive sexuality education is available & \\
\hline Gender Equality Goal Target 5.6 & $\times$ \\
Gender Equality in SRHR & \\
- Respect for women's sexual autonomy within marriage & \\
- Whether universal access to contraceptive and SRH information and services is & $\times$ \\
\hline & included in national policy
\end{tabular}

Notes Column headings are as follows: (1) available, (2) aspirational. * Currently available only in a small number of countries with special surveys. $\dagger$ Available for very few countries for those aged 10-14. Adapted from Guttmacher Institute $(2015$, p. 3).

istics and criticalities that had an impact on the epidemiological survey carried out by analyzing the Hospital Demission Records (HDR) provided by the IRCCS Burlo Garofolo. In the following, we will thus comment on some of the fields contained in the Excel files received, together with the technicalmethodological choices adopted to cope, where possible, to some critical issues encountered.

The basic information on the demission division of the patient highlighted a problem that must be taken into account, wanting to use this variable in a longitudinal analysis. A complex structure sometimes provides internal reorganizations and this may force interventions on past data. ${ }^{7}$ While the incorporation of a previously autonomous divisions can be easily manages, when part of a structure becomes autonomous we are faced with a more complex problem to face. Even more complex is the situation when hospital admissions become outpatient services, since information to analyze move from the hospital demission records to the outpatient services archive. In our case, this happened when the Medical Assisted Reproduction Division was abolished in 2014 and their activity passed to the outpatient services.

During a meeting with the Burlo Hospital partners, it was also learned that in some periods of time patients were admitted to the two main divisions (Obstetrics and Gynecology) taking into account organizational and not only diagnostic aspects. Hence the consequence that Hospitalization Division is a

\footnotetext{
${ }^{7}$ The situation is similar to that of the change from national currencies to Euro, which forces the conversion of past economic data into the new currency to allow analyses in a longitudinal perspective.
} 
variable to be used with caution in order to select the case studies of interest, resulting more reliable, to avoid the risk of losing relevant cases, the use the fields reporting the diagnoses and interventions performed on patients.

The database also reports the 'Progressive HDR', which shows an anomaly: starting from the first patient of the year, each admission is assigned an incremental HDR code, but the highest code number does not correspond to the total number of hospitalizations. The analysis of 'missing' HDR records shows that they tend to grow over time, reaching over $10 \%$ in the last two years. There are also some inexplicable oscillations, such as the sudden upsurge in 2007 and the halving in 2012. By ordering HDR records inside each year, one may note that the lack of HDR codes does not present a discernable 'pattern.' The deleted cards may be related to a demission followed by admission to another Department, although a new HDR should only be created in a few wellspecified cases. In fact, according to the prescriptions of the Ministry of Health: 'the identification number, attributed to each medical record and of the relative HDR, must be the same for the entire duration of admission, regardless of internal transfers within the same health institution. Exceptions are the transitions from ordinary to day hospital and from acute to rehabilitation or long-term care, or vice versa. In the latter cases, a new medical record and a new HDR must be completed' (Ministero della Salute, 2018, p. 10). One may guess that the removal of numerous HDRs depends on controls that detected duplications or induced to delete incomplete HDR cards, but it is necessary that these aspects are clarified in order to allow a correct use of this information.

Marital status, education and profession variables are present in the database, but unusable because in most cases the fields are not filled out. It is not clear why, given that marital status and education are classified as compulsorily fields in the Functional Specifications of the Ministry of Health (Ministero della Salute, 2017). These provisions are recent, but they do no more than reiterate that marital status is a mandatory field, since the introduction of the HDR with the D.M. October 27, 2000, n. 380, and education has been defined mandatory with the D.M. July 8, 2010, n. 135. Unfortunately, the unavailability of this information for the vast majority of hospitalizations deprives INTEGRA Project of important cognitive elements that would be useful for socially identifying hospitalized women and for the construction of the Sexual and Reproductive Health Index. Moreover, these shortcomings make it difficult to compare with the hospitalization analyzes carried out by Slovenian partners, who can count on the availability of complete information regarding the education and marital status of patients. 
Unfortunately, these deficiencies are generalized, to the point that the Ministry of Health summary of the history of the introduction of the HDR and the characteristics of this important tool ends with the paragraph 'Limitations and cautions in the use of data,' thus recognizing the existence of the problem. 'It is necessary to keep in mind that the HDR database, even if it has a very rich information content and an almost total coverage of the Italian hospitals, has limits and criticalities in the use, such as problems of homogeneity of compilation, completeness and accuracy for some variables (mainly in the first years) and variation of the classification systems over the years. ${ }^{8}$

In addition to incompleteness of information, data analysis showed an even more worrying aspect. Information about marital status and education, in addition to being available for only about a third of hospitalizations, revealed serious inconsistencies that are not acceptable because they undermine the reliability of the database. For example, crossing education and marital status with age, underage patients with a university degree were noticed and less than ten years old girls turn out to be married and sometimes even already separated. If there were few cases, we could think of inattention in writing data on paper or when recording on electronic media, but dozens of inconsistencies of this type suggest that they may also due to some kind of systematic error.

Faced with a rather generalizes situation, according to the Ministry of Health, it may be appropriate that the INTEGRA project guidelines will contain a strong recommendation to support the completeness and correctness of the compilation of the HDRs. The HDR database contains also fields in which up to six different diagnoses may be recorded. Specific diagnoses are very numerous and detailed, but they can be grouped into a smaller number of categories, identified by the first 3 digits of the codes used for registration. These categories may then be further grouped in 17 chapters, according to the International Classification of Diseases ICD-9-CM. Another six fields are reserved for the interventions to which the patient has been submitted during the hospitalization and interventions too may be aggregated, for the purposes of the analysis, in 17 general categories.

The plurality of recordable diagnoses and interventions caused a shortcoming detected by the analysis of the database that revealed the frequent duplication of similar diagnoses within the same HDR card. This appears to be

\footnotetext{
${ }^{8}$ See http://www.salute.gov.it/portale/temi/p2_6.jsp?id=1232\&area=ricoveriOspedalieri\&menu =vuot.
} 
imputable to the attribution of a general code together with a more specific code to the same diagnostic situation. In the case of childbirths, a frequent situation is that of the diagnosis ' 650 - Normal birth' coupled to the ' $V_{270}-$ Simple birth: born alive' diagnosis.

The problem could be solved by using the 'DRG - Diagnosis Related Group' code, which assigns the reasons for admission to a single category by identifying, using an algorithm, the 'prevalent diagnosis.' However, this may result in the loss of essential information, because a diagnosis, relevant to the construction of the Health Index, may not be 'intercepted' due to the attribution of that hospitalization to a DRG that does not appear to be significant.

Other anomalies found in the data may depend on errors in the registration of information or on an uneven application of the criteria for registration by the plurality of operators who contribute to the compilation of HDR cards. In any case, as we learn from the most recent Annual Report on Hospitalization in the section 'Completeness and Quality of the HDR Survey' (Ministero della Salute, 2017), compilation errors significantly affect the quality of the recorded information. The consequences of this situation are not trivial, as claimed by Roberto Vattovani (2009) in his doctoral thesis 'Italian-Slovenian Hospital Health Mobility after Slovenia's Accession to the European Union:' 'Even if HDRs do not have a strictly epidemiological purpose, being part of an administrative-accounting processes, the analysis of admissions by diagnosis provides a broad and articulated picture of hospitalization, a phenomenon of great importance for Public Health, both for the importance of the pathologies for which patients access this service, and for the considerable financial commitment that the various local health authorities dedicate to hospitals' (p. 25).

The author adds that the Ministry of Health, differently from the FVG Region, usually does not provide the individual HDR records, but only data aggregated according to some relevant attributes, thus limiting the execution of possible analyzes. For example, not knowing the place of residence of Slovenian citizens, the actual extent of the phenomenon of international health mobility cannot be estimated, even if this information is partially derivable through the table on hospitalization charges (Vattovani, 2009, p. 116). Even if this is a rather specific example, it is useful to underline the need to be able to count on detailed information at the 'micro' level, in order to carry out analyzes aimed at tasks such as the construction of a health index. In fact, while performing some aggregation towards the 'macro' level is possible, it is difficult to go down from the macro level to minute group segmentations, and impossible to reach the individual level. 


\section{From Hospitalizations to the Patients}

Since patients may repeatedly resort to hospital care, to estimate the number and type of user it is necessary to pass from data on admissions to a database in which the unit of analysis is the patient. After recording in the new database the number of admissions, it should be decided which other information contained in the 'demission' database should be associated with each patient. The procedure does not present any problems for the characteristics who remain stable over time, which are however few because only the place and date of birth certainly remain unchanged.

In case of multiple admissions, in addition to age, citizenship, residence, marital status, department of demission, diagnoses, interventions, etc. may also change. It is therefore necessary to decide whether to register, for example, citizenship at first or last admission, but it may be advisable to register both because, by comparing the two situations, one can obtain the useful additional information of a possible change of citizenship.

Similarly, you can record the year of the first, last or all admissions but, working in a longitudinal perspective, the choice between producing tables in which patients are 'anchored' to the first, or last, year is not neutral. In the first instance, there will be an artificial decline in patients over time: for example, a patient 'assigned' to 2006 (first admission), will not appear in the following years. On the contrary, assigning patients to the year of the last hospitalization, going back in time we will be confronted with an artificial rarefaction of patients.

In both cases, distortions do not allow an effective longitudinal analysis. Therefore, it seems reasonable to propose a different solution: patients are counted only once in case of multiple admissions in the same year and reinserted it in the database if admitted in a different year. Moreover, while aggregating the hospitalizations carried out in any wider period needs some kind of 'justification' (could be longer or shorter), year by year solution is supported by the Ministry of Health, using it for the Annual Report on Hospital Admission (Ministero della Salute, 2017) and hospital facilities too yearly allocate resources, report activities, etc.

Even this solution, however, presents a criticality, because it does not put all patients on the same level. In fact, about patients admitted at the beginning of the year we know the subsequent 'hospital history', but any previous admission is not detected; about patients admitted at the end of the year we know if they have already been hospitalized, but we cannot know what will happen in the future. In practice, if you want to study the hospital history 
of each patient in a year, once identified for each patient a referral hospitalization, from that date one should add to the clinical history of that patient all the possible hospitalizations occurred in the six months before and after. Similarly, extending the observation period to two years, starting from the 'central' admission date, the observation should be extended to the previous and following 365 days.

\section{Conclusions}

The Italian Parliamentary Commission of Inquiry into the System of Reception, Identification and Expulsion, as Well as the Conditions for the Detention of Migrants and Public Resources Committed, approved a Report on the Protection of the Health of Migrants and of the Resident Population on 8 November 2017 (Camera dei Deputati, 2017) which includes an analysis of the 'epidemiological situation' with which the conditions are laid for improving the health system in relation to the situation of subjective vulnerability of the migrants. Quite interesting, for the purposes of this work, is to cite the epidemiological analysis, stating that: 'Salgari Syndrome, i.e. the stereotype of the migrant "infector," a dangerous source of diseases, namely of infectious type, is supported today by no scientific evidence derived from formal epidemiological studies or from health surveillance. [...] On the contrary, the data on the health of migrants derived from studies and surveillance experiences unexpectedly outline a population exposed to the dangers of marginality. It deals of the so-called "exhausted migrant effect," which implies the more or less rapid depletion of the health patrimony of migrants, regardless the situation on their arrival, because of the continuous exposure to risk factors, due to the conditions of poverty in which they live' (Camera dei Deputati, 2017, p. 28).

The Report explicitly refers to the Strategy and Action Plan for Refugee and Migrant Health in the WHO European Region and to the homonymous resolution, approved on December 15, 2016. As for some aspects relevant to the INTEGRA project, the WHO document underlined that migrant women may often wish to be treated by female doctors, based on a principle of greater closeness, cultural sensitivity and gender equity. The document also speaks of an important effort of 'promotion of health literacy' (Camera dei Deputati, 2016, p. 40).

The Conclusions of the Report state: 'Among the priorities to be addressed is also that of a selection of the categories most in need of assistance. If it is true that migrants are in themselves a weak category, it is also true that there are categories in greater difficulty, the weak among the weak. These are those that the legislator defines "vulnerable categories." From the point of 
view of healthcare, attention paid to those most in difficulty means particular attention to issues such as sexual and reproductive health, family planning, gender-based violence and rape (linked to important sociocultural phenomena, such as forced marriage and teenage pregnancy), mental health, people suffering from trauma or injury, among which victims of torture are of particular importance' (Camera dei Deputati, 2017, pp. 72-73).

Quite evidently, the Conclusions of the Italian Parliament Commission legitimize the attempt to construct a Sexual and Reproductive Health Index, based on valid and reliable indicators.

\section{References}

Camera dei Deputati. (2017). Relazione sulla tutela della salute dei migrantie della popolazione residente (8 novembre 2017) della Commissione parlamentare di inchiesta sul sistema di accoglienza, di identificazione ed espulsione, nonché sulle condizioni di trattenimento dei migranti e sulle risorse pubbliche impegnate. Rome, Italy: Author.

Canadian International Development Agency. (1996). Guide to gender-sensitive indicators. Ottawa, Canada: Author.

Drost, E. A. (2011). Validity and reliability in social science research. International Perspectives on Higher Education Research, 38(1), 105-124.

Guttmacher Institute. (2015). Sexual and reproductive health and rights indicators for the SDGs post-2015 recommendations. New York, NY: Author.

Ministero della Salute. (2008). Classificazione delle malattie, dei traumatismi, degli interventi chirurgici e delle procedure diagnostiche e terapeutiche. Rome, Italy: Author.

Ministero della Salute. (2017). Rapporto annuale sull'attivitá di ricovero ospedaliero. Rome, Italy: Author.

Ministero della Salute. (2018). Specifiche funzionali. Rome, Italy: Author.

Moreland, S., \& Curran, J. (2018). A guide for monitoring and evaluating population-health-environment programs (2nd ed.). Chapel Hill, NC: University of North Carolina.

United Nations. (1995). Report of Fourth World Conference on Women. New York, NY: Author.

Vattovani, R. (2009). La mobilitá sanitaria ospedaliera italo-slovena dopo l'adesione della Slovenia all'Unione Europea (Unpublished PhD thesis). University of Trieste, Trieste, Italy.

World Health Organization. (1997). Selecting reproductive health indicators: A guide for district managers; Field testing version. Geneva, Switzerland: Author.

World Health Organization. (2006). Reproductive health indicators: Guidelines for their generation, interpretation and analysis for global monitoring. Geneva, Switzerland: Author. 
World Health Organization. (2016). Strategy and action plan for refugee and migrant health in the WHO European Region. Geneva, Switzerland: Author.

S. Ličen, I. Karnjuš, \& M. Prosen (Eds.). (2019). Women, migrations and health: Ensuring transcultural healthcare (pp. 59-72).

Koper, Slovenia: University of Primorska Press.

https://doi.org/10.26493/978-961-7055-43-6.59-72 